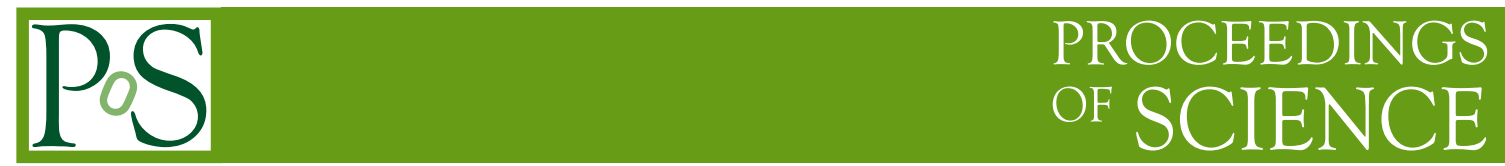

\title{
Towards the measurement of charged hadron spectra in CMS
}

\section{Ferenc Siklér* for the CMS Collaboration}

KFKI Research Institute for Particle and Nuclear Physics, Budapest, Hungary

E-mail: sikler@rmki.kfki.hu

The CMS experiment will provide good quality measurements of yields and spectra of identified charged and neutral particles, thus contributing to the study of soft hadronic physics at the LHC energies. The methods and tools necessary for producing charged hadron spectra in proton-proton collisions at $\sqrt{s}=14 \mathrm{TeV}$ are discussed.

High- $p_{T}$ Physics at LHC - Tokaj'08

March 16 - 192008

Tokaj, Hungary

${ }^{*}$ Speaker. 


\section{Introduction}

The CMS experiment at the LHC is a general purpose detector designed to explore physics at the $\mathrm{TeV}$ energy scale [1]. It has a large acceptance and hermetic coverage. The various subdetectors are: a silicon tracker with pixels and strips $(|\eta|<2.4)$; electromagnetic $(|\eta|<3)$ and hadronic $(|\eta|<5)$ calorimeters; and muon chambers $(|\eta|<2.4)$. The acceptance is further extended with forward detectors: CASTOR $(5.2<|\eta|<6.6)$ and Zero Degree Calorimeters $\left(\left|\eta_{\text {neutrals }}\right|>8.3\right)$. CMS detects leptons and both charged and neutral hadrons. In the following the soft physics capabilities are described. For an extensive review see Ref. [1].

One of the first physics results from the LHC will be the measurement of charged hadron spectra in p-p collisions. The measurement of these basic observables will also serve as an important tool for the calibration and understanding of the CMS detector and will help establishing a solid basis for exclusive physics. This example analysis uses 2 million inelastic p-p collisions. They have been generated by the PYTHIA event generator [2].

The minimum bias trigger will be based on counting towers with energy above the detector noise level, in both forward hadronic calorimeters (HF, $3<|\eta|<5$ ). A minimal number of hits (1, 2 or 3) will be required on one or on both sides, an energy threshold value of $1.4 \mathrm{GeV}$ is used in the hit definition [3]. Once the luminosity is high enough, events can also be taken with the so called zero-bias trigger based on a random clock each bunch crossing.

\section{Charged particle tracking}

Both pixel and strip silicon tracker detectors are used for the reconstruction of charged particles. While the procedure presented here roughly follows the standard one $[4,5]$, there are important differences and additions, since the software had to be adapted to the needs of minimum bias tracking. More specifically, the aim was to reconstruct as many particles and as many primary
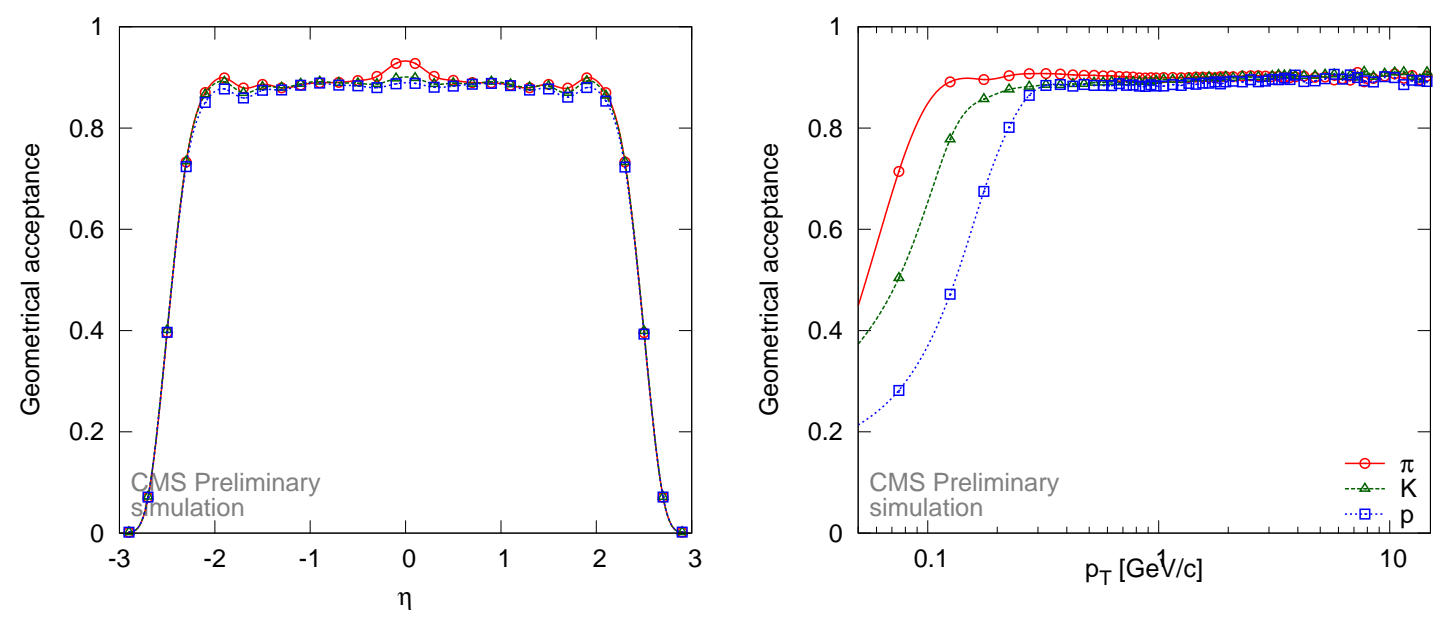

Figure 1: Geometrical acceptance as a function of $\eta$ (left) and as a function of $p_{T}$ (right). Values are given separately for pions (red circles), kaons (green rectangles) and protons (blue boxes). The curves are drawn to guide the eye. 
interaction vertices as possible, while keeping the quality and performance of the tracking at a high level. A large fraction of charged hadrons is produced with $p_{T}<1 \mathrm{GeV} / c$, the maximum of the $p_{T}$ spectra being well below $500 \mathrm{MeV} / c$. Thus, a good measurement of differential and integrated yields requires tracking down to as low $p_{T}$ values as possible. The extended $p_{T}$ reach is even more important for integrated yields, since extrapolations are model dependent, in particular in the low $p_{T}$ region.

Proto-tracks based on hit triplets were used. Requiring three hits instead of two (pair) is necessary to keep the rate of fake tracks very low at low $p_{T}$ or in events with many collisions (pile-up). With a modified hit triplet finding algorithm the pixel detector can be employed for the reconstruction of low $p_{T}$ charged particles $[1,6,7,8]$. The particles are restricted to originate from the vicinity of the beam-line. The minimal reconstructed $p_{T}$ is set to $0.075 \mathrm{GeV} / c$. The acceptance of the method extends down to $0.1,0.2$ and $0.3 \mathrm{GeV} / c$ in $p_{T}$ for pions, kaons and protons, respectively (Fig. 1). The obtained pixel tracks are used for finding and fitting the primary vertex or vertices [4]. The found vertices are reused, ensuring that the track comes from an interaction point and has at least three pixel hits. This brings the fake track rate down to per mille levels.

The measured shape in the case of pixel hits and the measured width in the case of strip hits are compared to the dimensions predicted from the local direction of the trajectory. This filter helps to eliminate incompatible trajectory candidates at an early stage. The seeds the trajectory building starts from are very clean, hence one seed is expected to produce only one global track. At the end, the trajectory is refitted with the primary vertex constraint. This last step ensures improved $\eta$ and $p_{T}$ resolution for low momentum particles.

\section{Corrections}

In general the corrections depend on the particle momentum, described by $\left(\eta, p_{T}, \phi\right)$, on the number of charged particles in the event, and on the number of pile-up collisions. The hadron spectra are corrected for particles of non-primary origin. Their main source is the feed-down from weakly decaying resonances, mostly $\mathrm{K}_{\mathrm{S}}^{0}, \Lambda / \bar{\Lambda}$ and $\Sigma^{+} / \bar{\Sigma}$. While the correction is around $2 \%$ for pions, it can go up to $15 \%$ for protons with $p_{T} \approx 0.3 \mathrm{GeV} / c$. The observed difference between pions and protons is expected because the daughter $\mathrm{p}$ or $\overline{\mathrm{p}}$ will take most of the momentum of the primary $\Lambda$ or $\bar{\Lambda}$ and therefore will more likely fit to the primary interaction point than a pion coming from a $\mathrm{K}_{\mathrm{S}}^{0}$ decay. The resonances $\mathrm{K}_{\mathrm{S}}^{0}, \Lambda$ and $\bar{\Lambda}$ can be extracted from the measured data, with help of V0 finding methods $[1,6,7,8]$. The direct measurement of their yield will make the feed-down correction much less dependent on simulation.

A recorded event is used in the analysis if it passes offline track or vertex triggers. While vertex finding is inefficient for few tracks, it is already efficient if there are more than 4 reconstructed tracks in the event. In the case of single inelastic events, the probabilities to reconstruct zero, one or two interaction vertices are $22 \%, 74 \%$ and $4 \%$, respectively. On average about 0.82 vertices are detected per event. According to this study, the presence of other vertices only slightly influences the reconstruction probability of a selected one. The efficiencies of the offline track trigger are: $88 \%$ inelastic, 99\% non-diffractive, 69\% double diffractive and 59\% single diffractive (as implemented in PYTHIA [2]). 

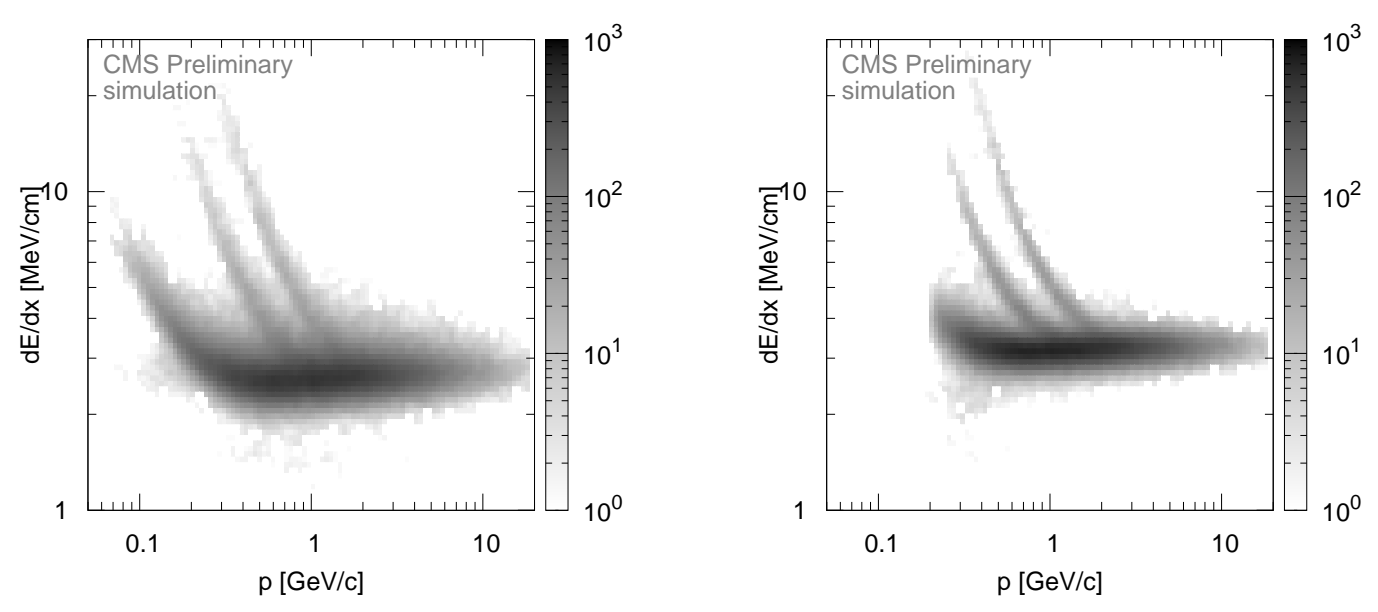

Figure 2: Distribution of the truncated mean estimator $\mathrm{d} E / \mathrm{d} x$ as a function of momentum $p$ for pixel hits (left) and strip hits (right).
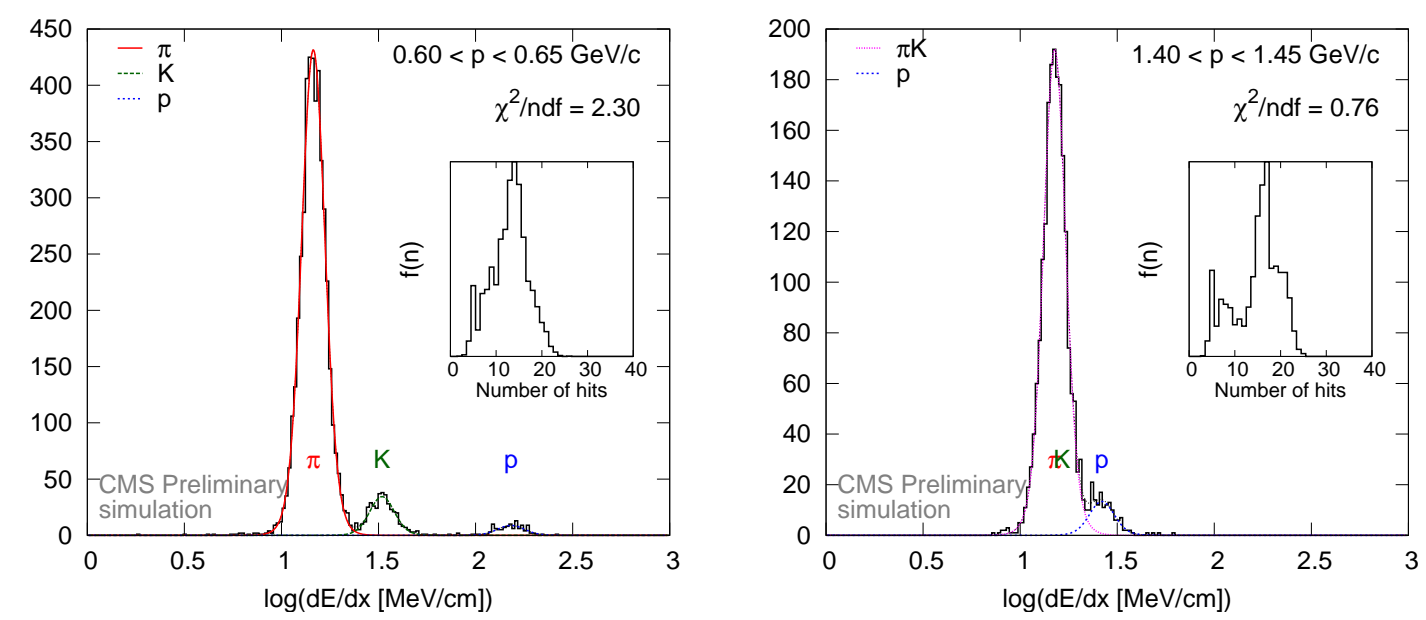

Figure 3: Example fits of $\log \mathrm{d} E / \mathrm{d} x$ histograms in several momentum bins. The distribution of the number of hits on track $f(n)$ is also given.

\section{Energy loss analysis}

Charged particles can be singly identified or their yields can be extracted (identification in the statistical sense) using deposited energy in the pixel and strip silicon tracker. Ten thousand minimum bias proton-proton events have been used in this study. The truncated mean estimator $\mathrm{d} E / \mathrm{d} x$, as a function of momentum is shown separately for pixel and strip hits in Fig. 2. The bands of pions, kaons and protons are well visible in the plots. The different momentum acceptances of the pixel and strip detectors can also be seen. Pixel and strip hits can only be combined if their 

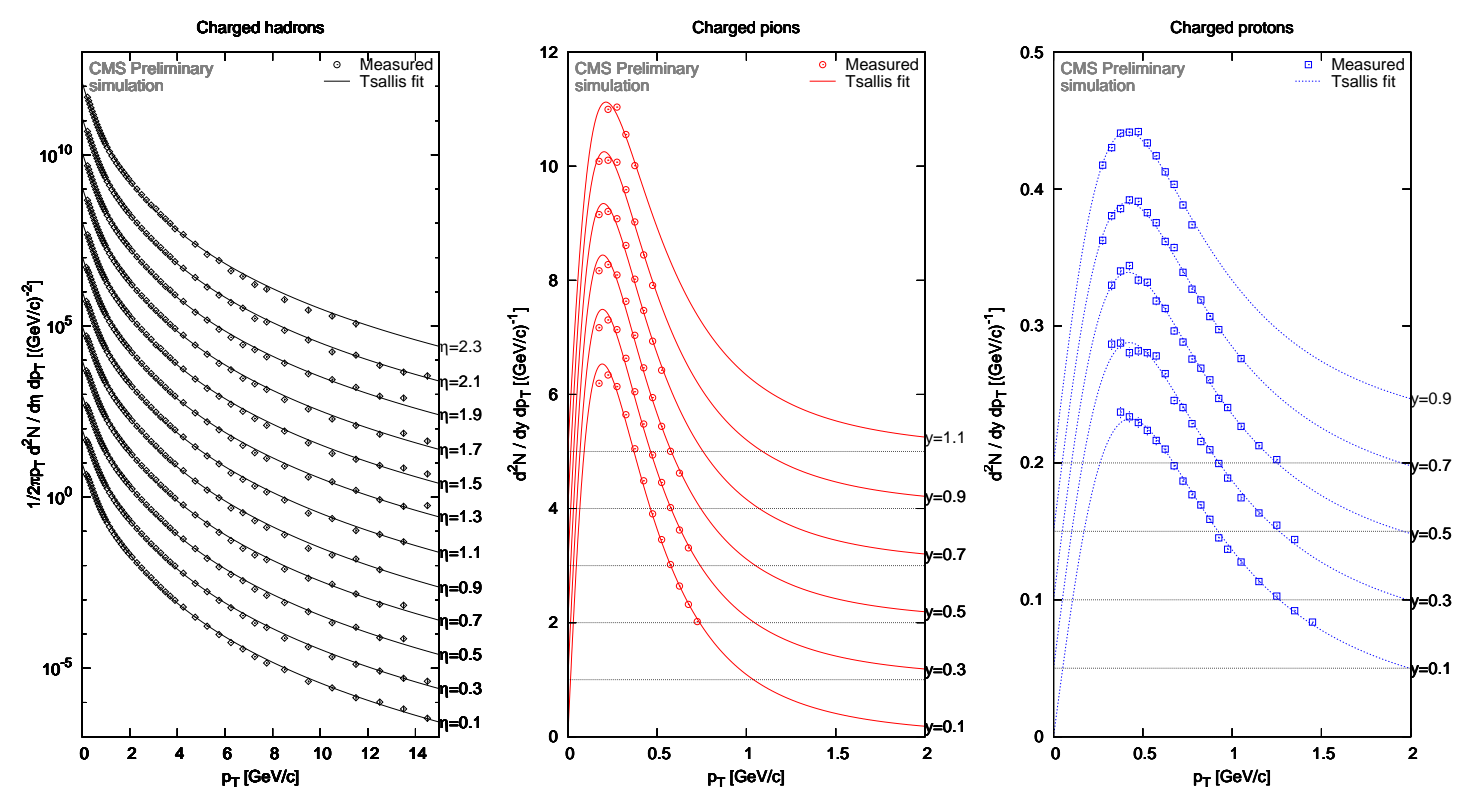

Figure 4: Selection of particle spectra. Left: Measured invariant yields of charged hadrons in the range $0<|\eta|<2.4$ with a series of 0.2 unit wide bins. Measured values and empirical fit functions are plotted. Values are successively multiplied by 10 for clarity. Right: Measured differential yields of identified charged pions and protons in the range $0<|y|<1.2$ with a series of 0.2 unit wide bins. Measured values and empirical fit functions are plotted. Values are successively shifted upwards for clarity. Only those rapidity bins are used where there are at least four measured points.

values are normalized to each other.

The distribution of $\log \mathrm{d} E / \mathrm{d} x$ is close to gaussian, its resolution being inversely proportional to the square root of the number of hits, $\sigma(n)=\sigma_{0} / \sqrt{n}$. The distribution of $\log \mathrm{d} E / \mathrm{d} x$ can be successfully fitted in slices of momentum. The fit function is a sum for particle species: here pions, kaons and protons are assumed. The effects of the momentum resolution and of the finite momentum window can also be taken into account. A fit with three species has 9 parameters: three yields, three means and three standard deviation values. Example fits in several momentum windows are given in Fig. 3. The relative resolution of $\mathrm{d} E / \mathrm{d} x$ for tracks with average number of hits $(\sim 15)$ is around 5-7\%. The contribution of different particle species can be unfolded if their means are well separated, with respect to the average resolution. The yield of kaons can be extracted if $p<0.8 \mathrm{GeV} / c$ and that of protons if $p<1.5 \mathrm{GeV} / c$. Both limits correspond to approximately $3 \sigma$ separation.

\section{Results}

In this analysis only measurements from those $\left(\eta, p_{T}\right)$ bins are used where the geometrical acceptance is above $50 \%$, the algorithmic efficiency is above $75 \%$, the fake track rate is below $10 \%$, multiple track counting is below $10 \%$, and the feed-down correction is below $25 \%$. This limits the kinematical region to $|\eta|<2.4$ and $p_{T}>0.15 \mathrm{GeV} / c$ for charged hadrons. 

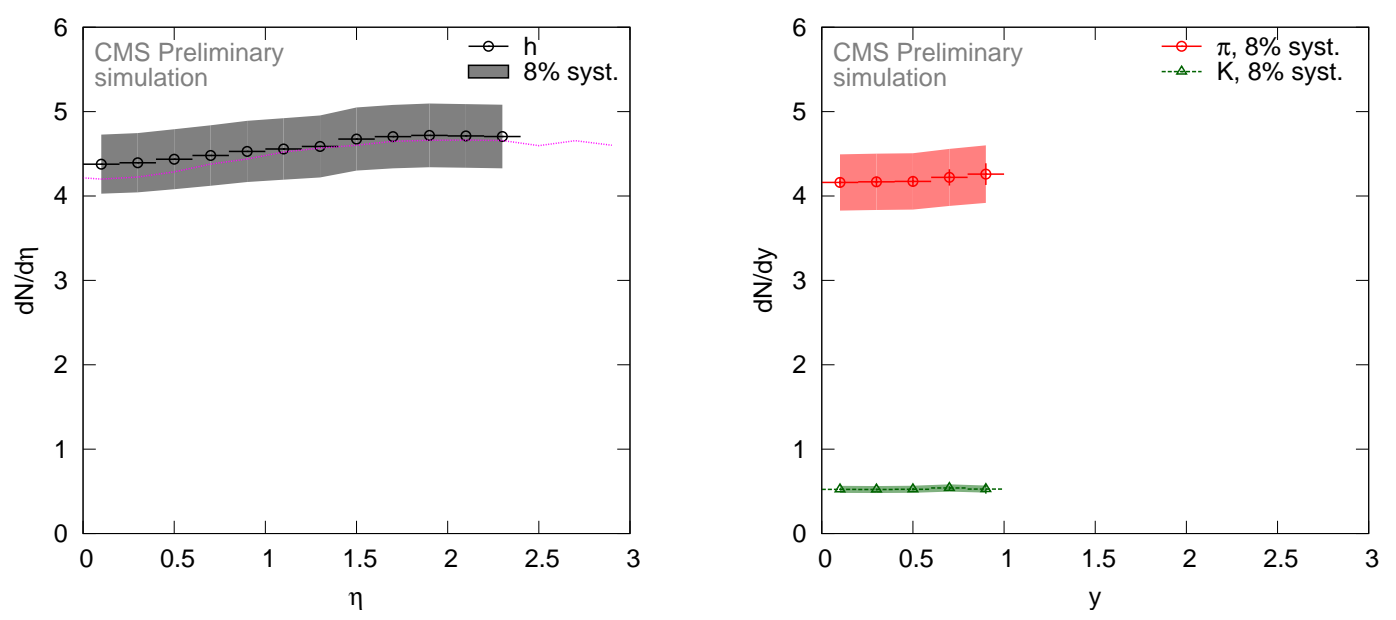

Figure 5: Left: Pseudorapidity density distribution of charged hadrons. The distribution from simulated tracks is given by the purple curve for comparison. Right: Rapidity distribution of charged pions and kaons. Estimated systematic error bands $(8 \%)$ are also shown.

The invariant yields were fitted by the Tsallis function [9], a function that successfully combines and describes both the low $p_{T}$ exponential and the high $p_{T}$ power-law behaviors:

$$
E \frac{d^{3} N}{d p^{3}}=\frac{d N}{d y} \frac{(n-1)(n-2)}{2 \pi n T[n T+(n-2) m]}\left[1+\frac{E_{T}\left(p_{T}\right)}{n T}\right]^{-n}
$$

where $E_{T}\left(p_{T}\right)=\sqrt{m^{2}+p_{T}^{2}}-m$. This form provides both the inverse slope parameter $T$, characteristic for low $p_{T}$, and the exponent $n$, of the high $p_{T}$ power-law tail. The average transverse momentum is $\left\langle p_{T}\right\rangle=2 n /(n-3) \cdot T$.

In general, results refer to the sum of positively and negatively charged particles. Symmetric positive and negative $\eta$ bins are also added. The measured invariant yields of charged hadrons are shown in Fig. 4-left, as a function of $p_{T}$ and in narrow $\eta$ bins (0.2 units, in the range $\left.|\eta|<2.4\right)$. Measured differential yields of identified charged pions and protons are shown in Fig. 4-right. The fitted parameters change only slightly with $\eta$. The inverse slope parameter is $T \approx 0.2 \mathrm{GeV} / c$, the exponent of the high $p_{T}$ power-law tail is $n \approx 7.2$. The average transverse momentum is $\left\langle p_{T}\right\rangle \approx$ $0.7 \mathrm{GeV} / c$.

The integral of the fit function is $d N / d y$, therefore giving direct access to the integrated yield. However, in the case of unidentified charged hadrons the fits in the low $p_{T}$ region are not very good. Their yield can be obtained by integrating the fit function for $p_{T}>2 \mathrm{GeV} / c$, summing the measured differential yields for $0.2<p_{T}<2 \mathrm{GeV} / c$ and adding a correction for $p_{T}<0.2 \mathrm{GeV} / c$. The pseudorapidity distribution of charged hadrons (Fig. 5-left) and the rapidity density distributions of pions and kaons are shown in Fig. 5-right.

The energy dependence of some measured quantities can also be studied. The pseudorapidity density of charged hadrons at $\eta \approx 0$ follows the trend from lower energies, which is linear in $\log \sqrt{s}$ (Fig. 6-left). While the average transverse momentum of charged hadrons at lower energies 

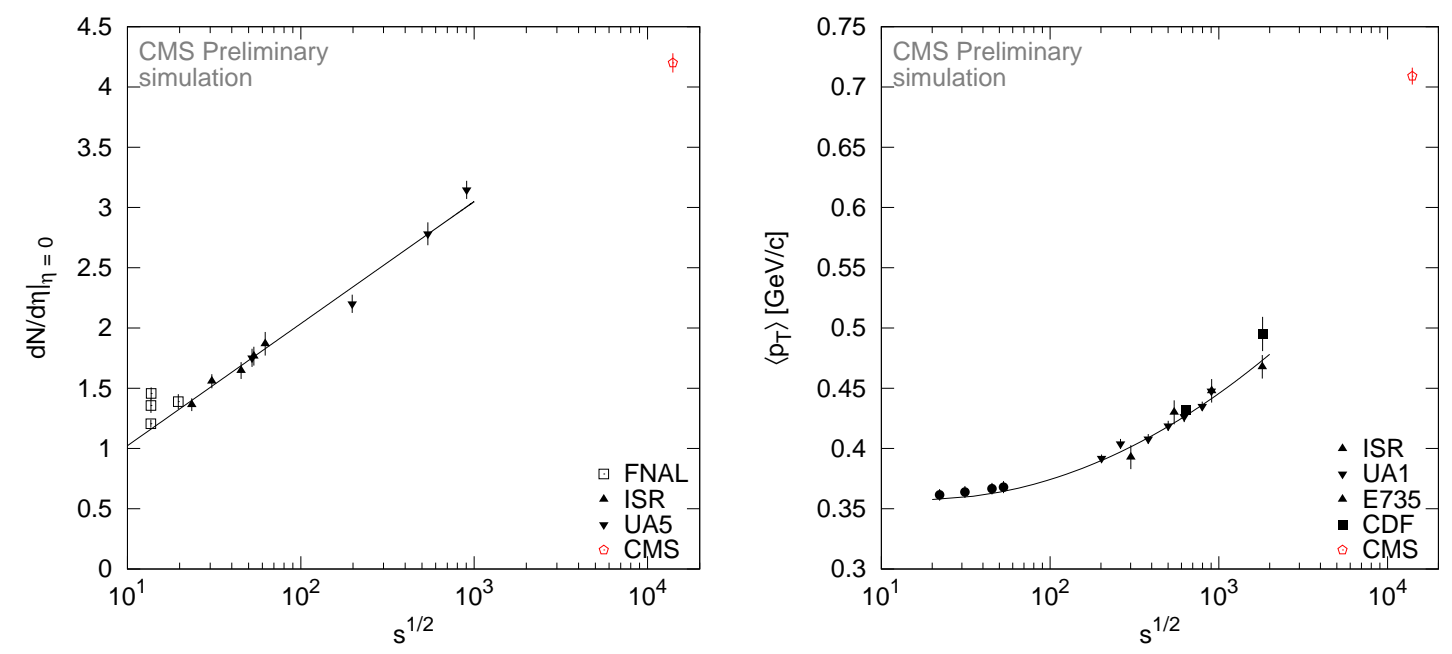

Figure 6: Left: Energy dependence of pseudorapidity density of charged hadrons at $\eta \approx 0$. The line shows the fit on data points of other experiments [10] in the form $0.01+0.44 \log \sqrt{s}$. Right: Energy dependence of average transverse momentum of unidentified charged hadrons at $\eta \approx 0$. The curve shows the fit on data points of other experiments [11] in the form $0.40-0.03 \log \sqrt{s}+0.0053(\log \sqrt{s})^{2}$. Results of this analysis are shown with red pentagons.
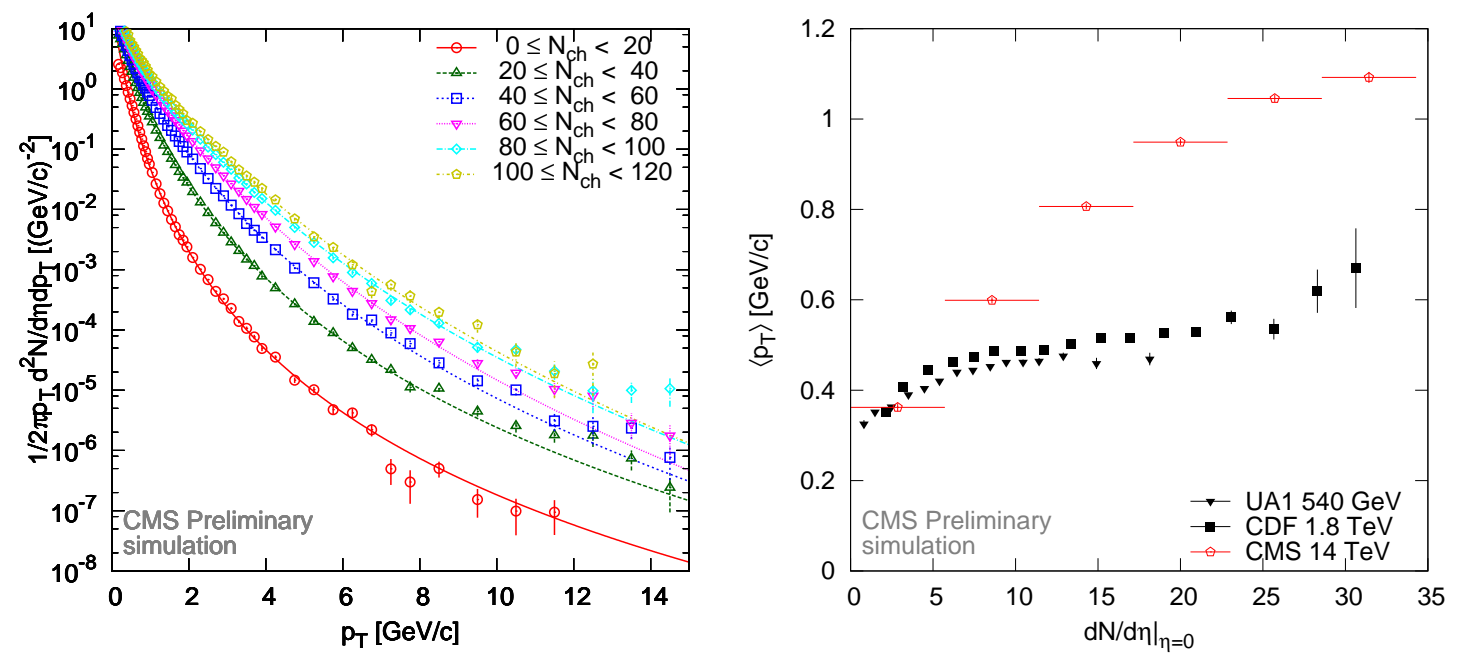

Figure 7: Multiplicity dependence. Left: measured invariant yields of charged hadrons at $-0.2<\eta<0.2$ for several multiplicity bins (number of reconstructed charged particles $N_{c h}$ ). Measured values and empirical fit functions are plotted. Right: average transverse momentum $\left\langle p_{T}\right\rangle$ at $\eta \approx 0$ as a function of rapidity density of charged particles $d N /\left.d \eta\right|_{\eta=0}$ with a comparison to UA1 [12] and CDF results [13].

is described by a quadratic function in $\log \sqrt{s}$, the result points to an accelerated increase of $\left\langle p_{T}\right\rangle$ when going to LHC energies (Fig. 6-right).

The multiplicity dependence of the charged hadron $p_{T}$ distributions is shown in Fig. 7-left. The 
multiplicity bins are given with the number of reconstructed charged particles $N_{c h}$. The distribution is the steepest for the lowest multiplicity bin and gets flatter with increasing $N_{c h}$. This is reflected in Fig. 7-right, which shows the $\left\langle p_{T}\right\rangle$ as a function of charged particle density at midrapidity.

\section{Conclusions}

Cross-sections and differential yields of charged particles (unidentified and identified pions, kaons and protons), produced in inelastic proton-proton collisions at $\sqrt{s}=14 \mathrm{TeV}$, can be measured with good precision with the CMS tracker. This analysis opens the door to several follow-up physics analyses: comparisons to theoretical models at low and high $p_{T}$; study of multiplicity distributions; studies of multiplicity and energy dependence of mid-rapidity density and average transverse momentum.

\section{Acknowledgment}

The author wishes to thank the Hungarian Scientific Research Fund and the National Office for Research and Technology (K 48898, H07-B 74296).

\section{References}

[1] D. d'Enterria et al., CMS physics technical design report: Addendum on high density QCD with heavy ions, J. Phys. G 34 (2007) 2307.

[2] T. Sjostrand, S. Mrenna and P. Skands, PYTHIA 6.4 physics and manual, JHEP 0605 (2006) 026.

[3] R. S. Hollis, A. Iordanova, J. Callner and D. J. Hofman, Zero bias and HF-based minimum-bias triggering for pp collisions at $14 \mathrm{TeV}$ in CMS, CMS PAS QCD-07-002.

[4] S. Cucciarelli, M. Konecki, D. Kotliński and T. Todorov, Track reconstruction, primary vertex finding and seed generation with the Pixel Detector, CMS Note 2006/026.

[5] W. Adam, B. Mangano, T. Speer, T. Todorov, Track Reconstruction in the CMS tracker, CMS Note $2006 / 041$.

[6] F. Sikler, Low p (T) hadronic physics with CMS, Int. J. Mod. Phys. E 16 (2007) 1819.

[7] F. Sikler [CMS Collaboration], Soft probes of high density QCD physics with CMS, J. Phys. G 35 (2008) 044045.

[8] The CMS Collaboration, Measurement of charged hadron spectra in proton-proton collisions at $\sqrt{s}=$ $14 \mathrm{TeV}, \mathrm{CMS} P A S$ QCD-07-001.

[9] C. Tsallis, Possible Generalization of Boltzmann-Gibbs Statistics, J. Statist. Phys. 52 (1988) 479.

[10] G. J. Alner et al. [UA5 Collaboration], Scaling of Pseudorapidity Distributions at c.m. Energies Up to 0.9-TeV, Z. Phys. C 33 (1986) 1.

[11] T. S. Ullrich, Experimental summary on global observables, hadron spectra and ratios, Nucl. Phys. A 715 (2003) 399.

[12] G. Arnison et al. [UA1 Collaboration], Transverse Momentum Spectra For Charged Particles At The Cern Proton Anti-Proton Collider, Phys. Lett. B 118 (1982) 167.

[13] T. Alexopoulos et al., Multiplicity dependence of the transverse momentum spectrum for centrally produced hadrons in anti-proton - proton collisions at $\sqrt{s}=1.8 \mathrm{TeV}$, Phys. Rev. Lett. 60 (1988) 1622. 